\title{
Coverage Control with Information Decay in Dynamic Environments
}

\author{
Nico Hübel ${ }^{*}$ S. Hirche ${ }^{* *}$ A. Gusrialdi ${ }^{* *}$ T. Hatanaka ${ }^{* *}$ \\ M. Fujita ${ }^{* *}$ O. Sawodny* \\ * Institute for System Dynamics, Universität Stuttgart \\ ** Fujita Laboratory, Dept. of Mechanical and Control Engineering, \\ Tokyo Institute of Technology
}

\begin{abstract}
In this paper a method for coverage control for a convex region $\mathcal{D} \subset \mathbb{R}^{2}$ in a dynamic environment is studied. An information map is introduced in which the information about each point is decaying with respect to time s.t. the robots must revisit them periodically. Also a timevarying density function is used for modeling moving points of interest. The considered gradient based control approach causes the cost function to stay within the desired bounds. But due to the non-stationary problem setup caused by the information decay it does not converge to a single point but to a bounded set, such that the robots keep gathering information continuously. With this method it is possible to gather information about several points of interest within the region $\mathcal{D}$ with only a few robots. In the end simulation results are presented to outline the effectiveness of the proposed control law.
\end{abstract}

\section{INTRODUCTION}

Natural disasters with their need for quick humanitarian help as well as military and surveillance operations and tasks in hazardous environments are major application for robots. In these kind of applications we often have to deal with additional difficulties like an adversarial environment, constraints (e.g. time restrictions, limited communication capabilities, etc.) and changing mission objectives. But for the use of robots in these scenarios they also must be economically reasonable. This is the motivation for the approach of this paper to use only a few robots, which should gather information in a specified area.

Recently a lot of research results came up for the area of coverage control. A very nice introductory overview can be found in Martínes [2007]. Most of the results rely on Voronoi tessellations. For example in Cortés [2004] a gradient descent to reach the optimal Voronoi configuration is proposed and in Martínes [2004] coverage control algorithms for robot groups with limited-range interactions are presented. Other results use some explicit information measures to express a gain of information. For instance Olfati-Saber [2007] proposes an algorithm which causes mobile agents with a dynamic network topology to improve their estimation of a moving target. In Martínez [2005] motion coordination algorithms which maximize the determinant of a Fisher Information Matrix are presented and in Basir [1995] a solution to an active sensing task is given which minimizes the variance of the estimation error and thus reduces the uncertainty of the target state. In addition there are also results like Ahmadzadeh [2007] which are based on receding horizon control or MPC.

In this paper the problem of gathering information and monitoring an area with only a few robots is addressed. The aim was to derive results, which are applicable for infinite time and therefore not converge to a single point but to a bounded set. In order to achieve that, an idea similar to the effective coverage function in Hussein [2007] but with the novel concept of an information model with information decay is introduced.

The paper is organized as follows. In section 2 the problem setup and the necessary definitions are presented and explained. Then the control law will be introduced and discussed in section 3 . The results were validated by extensive simulations. In section 4 a simulation for covering a square area with a moving point of interest is presented before a conclusion and the possible extensions of this work are stated in section 5 .

\section{PROBLEM SETUP}

In this paper $Q=\mathbb{R}^{2}$ denotes the configuration space of the agents. Let $\mathcal{D}$ be the area which should be monitored by the agents. $\mathcal{D}$ must be a convex subset of $Q$. Throughout the paper $\mathbb{R}^{+}=\{a \in \mathbb{R} \mid a \geq 0\}$ is used.

\subsection{Agent model}

Let $\mathcal{A}=\left\{\mathcal{A}_{i} \mid i \in S=\{1,2,3, \ldots, N\}\right\}$ be the set of agents consisting of the single agents $\mathcal{A}_{i}$ with $N$ being the number of agents and $S$ being the index set of the fully connected network which means that it contains the indices of all agents. Let $\mathbf{q}_{i} \in Q$ denote the position of agent $\mathcal{A}_{i}$. All agents $\mathcal{A}_{i}$ satisfy the kinematic equation

$$
\dot{\mathbf{q}}_{i}=\mathbf{u}_{i}, \quad i \in S
$$

with $\mathbf{u}_{i} \in \mathbb{R}^{2}$ as the control input of agent $\mathcal{A}_{i}$. It is assumed that the underlaying dynamics of the agents are controlled by low level controllers which use $\mathbf{u}_{i}$ as reference input.

\subsection{Measurement function}

The measurement function

$$
\mathcal{M}_{i}\left(s_{i}\right): \mathcal{D} \times Q \rightarrow \mathbb{R}^{+} \text {with } s_{i}=\left\|\mathbf{p}-\mathbf{q}_{i}\right\|^{2}
$$

of the agent $\mathcal{A}_{i}$ is defined as a $\mathcal{C}^{1}$-continuous map that describes the sensing performance of that agent. Sensing 
performance in this case means how much information the agent can get about a fixed point $\mathbf{p} \in \mathcal{D}$ depending on his own position. Naturally sensing performance is decreasing when the distance to the agent increases, which leads to the assumption of $\mathcal{M}_{i}$ being a monotonically decreasing function of the distance between a point $\mathbf{p} \in \mathcal{D}$ and the position of the robot $\mathbf{q}_{i}:\left.\frac{\partial \mathcal{M}_{i}}{\partial s_{i}}\right|_{s_{i}=\left\|\mathbf{p}-\mathbf{q}_{i}\right\|^{2}} \leq 0 \forall \mathbf{p} \in$ $\mathcal{D}$ and $\mathbf{q}_{i} \in Q \quad \forall i \in S$. Additionally the agent can only gather information in a certain area around his position. Therefore the sensor area of the agent $\mathcal{A}_{i}$ is defined as $\mathcal{W}_{i}=\left\{\mathbf{p} \in \mathcal{D} \mid\left\|\mathbf{p}-\mathbf{q}_{i}\right\| \leq r_{i}^{2}\right\}$ with the sensor range $r_{i}$. This leads to the following additional assumption on the measurement function: $\mathcal{M}_{i}\left(s_{i}\right)=\left.\frac{\partial \mathcal{M}_{i}}{\partial s_{i}}\right|_{s_{i}=\left\|\mathbf{p}-\mathbf{q}_{i}\right\|^{2}}=$ $0 \quad \forall \mathbf{p} \in \mathcal{D} \backslash \mathcal{W}_{i}=\left\{\mathbf{p} \mid s_{i} \geq r_{i}^{2}\right\}$. Now let the measurement map

$$
\mathcal{M}(\mathbf{s})=\sum_{i} \mathcal{M}_{i}\left(s_{i}\right) \forall i \in S
$$

be defined as the sum of all measurement functions and $\mathbf{s}$ be the set containing all $s_{i}$.

An example of such a measurement function is

$$
\mathcal{M}_{i}\left(s_{i}\right)= \begin{cases}\frac{C_{i}}{r_{i}^{4}}\left(s_{i}-r_{i}^{2}\right)^{2} & \text { if } s_{i} \leq r_{i} \\ 0 & \text { if } s_{i}>r_{i}\end{cases}
$$

which is also depicted in fig. 1 .

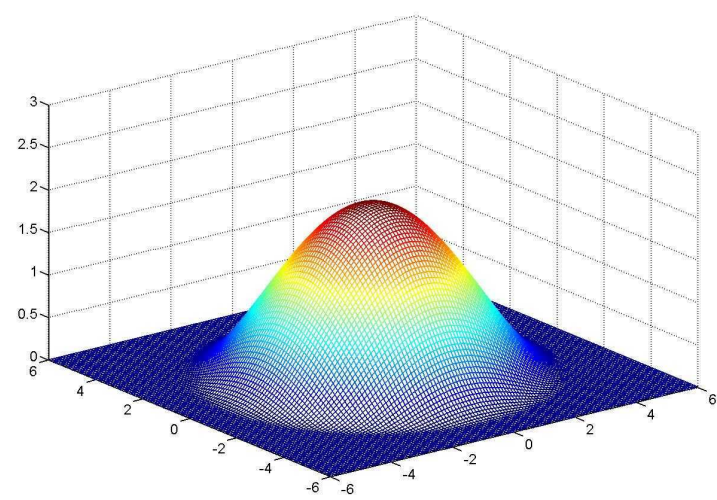

Fig. 1. Meassurement function (4) with $C_{i}=2$ and $r_{i}=5$.

\subsection{Information model}

Let the information map be defined as $I(\mathbf{p}, t): \mathcal{D} \times$ $\mathbb{R}^{+} \rightarrow \mathbb{R}^{+}$. The evolution of the map is modeled with the following partial differential equation:

$$
\frac{\partial}{\partial t} I(\mathbf{p}, t)=\delta I(\mathbf{p}, t)+\mathcal{M}(\mathbf{s})
$$

with the decay rate $\delta \leq 0$. The information map indicates how much information the agents have gathered about the points $\mathbf{p} \in \mathcal{D}$. It consists of two parts. The first part $\delta I(\mathbf{p}, t)$ represents the information decay whereas the information map $\mathcal{M}(\mathbf{s})$ in the second part represents the information gain. For $\delta<0$ the information is decaying over the time s.t. all the points have to be revisited frequently in order to keep the information level high. For $\delta=0$ the results of Hussein [2007] are a special case of the results presented in this paper.
In this method $\delta, I_{\text {ref }}(\mathbf{p})$ and $k_{i}$ are considered as design parameters. $I_{r e f}(\mathbf{p})$ is the reference information map which gives the level of information the robots should gather for each point of $\mathcal{D}$ and $k_{i}$ are the feedback gains. While according to (7) the feedback gains are directly related to the velocity of the robots, the choice of $\delta$ and $I_{r e f}(\mathbf{p})$ is more complicated. But according to (5) $\delta I_{\text {ref }}(\mathbf{p})+\mathcal{M}(\mathbf{0}) \geq 0$ should hold for all $\mathbf{p} \in \mathcal{D}$ because otherwise $I_{\text {ref }}(\mathbf{p})$ never can be reached in all points $\mathbf{p} \in \mathcal{D}$. An explicit relation between the speed of the robots and the design parameters will be the subject of further research.

\subsection{Density function}

Let $\phi(\mathbf{p}, t): \mathcal{D} \times \mathbb{R}^{+} \rightarrow \mathbb{R}^{+}$be the time-variant positive semi-definite density function which represents the regions of interest in the area $\mathcal{D}$. The robots will always spend particular interest on gathering information in regions where the density function has a high value and will gather information about adjacent regions only if they already have gathered enough information about that regions of interest. Moving points of interest such as the targets of a surveillance operation can be represented by the moving peaks of a time-variant density function. This will cause the robots to follow these peaks. There are two assumptions on $\phi(\mathbf{p}, t)$. First, it must be two times continuously differentiable with respect to time. This assumption is not very restrictive due to the fact that real points of interest also can not instantly change their position. And possible newly detected points of interest can be incorporated by a slowly rising peak. Second, $\frac{\partial \phi}{\partial t}(\mathbf{p}, t) \ll k_{i}$ and $\frac{\partial^{2} \phi}{\partial t^{2}}(\mathbf{p}, t) \ll k_{i}$ must hold which physically means that the speed and the acceleration of the target has to be small compared to the robot. In this paper the time-varying density function is assumed to be pre-known and the problem of online-estimation and adaption is not considered. But there are already existing results and algorithms available from the field of computer science.

\subsection{Cost function}

Let

$$
J(t)=\int_{\mathcal{D}} h\left(I_{r e f}(\mathbf{p})-I(\mathbf{p}, t)\right) \phi(\mathbf{p}, t) d \mathbf{p}
$$

be the cost function which should be minimized with the penalty function $h\left(e_{I}\right)$ which penalizes a lack of coverage which in addition is weighted according to the importance of the area denoted by the density function. There are some necessary assumptions on the penalty function $h\left(e_{I}\right)$ with $e_{I}=I_{\text {ref }}(\mathbf{p})-I(\mathbf{p}, t)$ :

$$
\begin{array}{ll}
\text { A1 } & h\left(e_{I}\right) \text { must be piecewise } \in \mathcal{C}^{1} \\
\text { A2 } h\left(e_{I}\right), \frac{\partial h}{\partial e_{I}}\left(e_{I}\right), \frac{\partial^{2} h}{\partial e_{I}^{2}}\left(e_{I}\right)>0, \quad \forall e_{I}>0 \\
\text { A3 } h\left(e_{I}\right), \frac{\partial h}{\partial e_{I}}\left(e_{I}\right), \frac{\partial^{2} h}{\partial e_{I}^{2}}\left(e_{I}\right)=0, \quad \forall e_{I} \leq 0
\end{array}
$$

A2 and A3 basically mean that only a lack of information is penalized but not having too much information. For instance the function $h(x)=\left(\max \left(0, e_{I}\right)\right)^{2}$ will be used for the simulation. 


\section{MAIN RESULT}

Now the control law is introduced which will cause the objective function (6) to stay bounded and its validity is proven by a Lyapunov function approach. In addition there will be a short physical interpretation of the problem and a relation between the decay rate $\delta$ and the achieved performance will be discovered. Then the theorem will be extended for partially or even non connected robot groups. The following abbreviations will be used:

$$
\begin{aligned}
h^{\prime}\left(e_{I}\right) & =\left.\frac{\partial h}{\partial e_{I}}\right|_{e_{I}=I_{\text {ref }}(\mathbf{p})-I(\mathbf{p}, t)} \\
h^{\prime \prime}\left(e_{I}\right) & =\left.\frac{\partial^{2} h}{\partial e_{I}^{2}}\right|_{e_{I}=I_{\text {ref }}(\mathbf{p})-I(\mathbf{p}, t)} \\
\mathcal{M}^{\prime}\left(s_{i}\right) & =\left.\frac{\partial \mathcal{M}_{i}}{\partial s_{i}}\right|_{s_{i}=\left\|\mathbf{p}-\mathbf{q}_{i}\right\|^{2}}
\end{aligned}
$$

Fully connected robot group: As a starting point a fully connected robot group is considered, which means that each agent can communicate with all other agents. Thus the index set is $S=\{1,2,3, \ldots, N\}$. Consider the following control law

$$
\mathbf{u}_{i}\left(\mathbf{q}_{i}\right)=-k_{i} \int_{\mathcal{W}_{i}} h^{\prime}\left(e_{I}\right) \mathcal{M}^{\prime}\left(s_{i}\right)\left(\mathbf{p}-\mathbf{q}_{i}\right) \phi(\mathbf{p}, t) d \mathbf{p}
$$

with the feedback gains $k_{i} \in \mathbb{R}^{+}$.

Please note that the input for each agent $\mathcal{A}_{i}$ only depends on his own position $\mathbf{q}_{i}$ explicitly. The information about the positions of the other robots are only used indirectly through the use of the information map $I(\mathbf{p}, t)$ in the penalty function. According to equation (5) only for the evolution of the information map the information about all robot positions is required. This corresponds to the concept of a 'world model' which often can be found in computer science literature on artificial intelligence.

Note further that it is not necessary to evaluate the integral over the whole area of $\mathcal{D}$. Because $M^{\prime}\left(s_{i}\right)=$ $0 \forall \mathbf{p} \notin \mathcal{W}_{i}$ holds by definition, it is sufficient to evaluate the integral over $\mathcal{W}_{i}$. While this reduces computational effort it causes the problem of local minima which will be solved later.

A closer look at the control law will reveal that it 'sums up' the weighted vectors from the robot to each point $\mathbf{p} \in \mathcal{W}_{i}$. The use of the gradient of the error distribution inside $\mathcal{W}_{i}$ causes the robot to move in the direction with the maximum error inside its sensor range. Through the following remark this can be seen easily.

Remark 1. Here some properties which are often used in mechanics but also hold for more general problems will be reviewed. For a region $V \subset \mathbb{R}^{n}$ and a generalized mass density function $\rho(p)$ with $p \in V$, the generalized mass and generalized center of mass is given as follows:

$$
\begin{aligned}
M_{V} & =\int_{V} \rho(p) d p \\
C_{V} & =\frac{1}{M_{V}} \int_{V} p \rho(p) d p .
\end{aligned}
$$

By splitting up the integral and using (8) and (9) with $V=\mathcal{W}_{i}$ and $\rho(p)=h^{\prime}\left(e_{I}\right) \mathcal{M}^{\prime} \phi(\mathbf{p})$ we find out that the control law (7) can also be expressed as follows:

$$
\mathbf{u}_{i}\left(\mathbf{q}_{i}\right)=-k_{i} M_{\mathcal{W}_{i}}\left(C_{\mathcal{W}_{i}}-\mathbf{q}_{i}\right)
$$

Note that the control law can become zero if $h^{\prime}\left(e_{I}\right)$ is equal to zero everywhere in $\mathcal{W}_{i}$ even if $\mathrm{J}(\mathrm{t})$ is not equal to zero. This happens if the following condition holds:

$$
I \geq I_{\text {ref }} \forall \mathbf{p} \in \mathcal{W}_{i} \stackrel{A 3}{\Rightarrow} h^{\prime}=0 \forall \mathbf{p} \in \mathcal{W}_{i} \stackrel{(7)}{\Rightarrow} \mathbf{u}_{i}\left(\mathbf{q}_{i}\right)=0
$$

To move the robot away from condition (11) the following simple linear control law is used

$$
\hat{\mathbf{u}}_{i}\left(\mathbf{q}_{i}\right)=-\hat{k}_{i}\left(\mathbf{q}_{i}-\hat{\mathbf{p}}_{i}\right)
$$

with the control gains $\hat{k}_{i} \in \mathbb{R}^{+}$. The only requirement on the point $\hat{\mathbf{p}}_{i}$ is that it has to drive the robots away from condition (11). How to choose $\hat{\mathbf{p}}_{i}$ specifically is not considered in this paper. But one possibility is to choose $\hat{\mathbf{p}}_{i}$ as the nearest point for which condition (11) does not hold. A more detailed discussion of the second control law can be found in the remark after the proof of the following theorem.

Theorem 1. Under the given assumptions the control law

$$
\mathbf{u}_{i}^{*}\left(\mathbf{q}_{i}\right)=\left\{\begin{array}{lll}
\mathbf{u}_{i}\left(\mathbf{q}_{i}\right) & \text { if } \quad h^{\prime} \neq 0 \text { for some } \mathbf{p} \in \mathcal{W}_{i} \\
\hat{\mathbf{u}}_{i}\left(\mathbf{q}_{i}\right) & \text { if } \quad h^{\prime}=0 \forall \mathbf{p} \in \mathcal{W}_{i}
\end{array}\right.
$$

with sufficiently large gains $k_{i}, \hat{k}_{i} \in \mathbb{R}^{+}$will hold the objective function within the bounds $C>J(t) \geq 0 \forall t>0$. Therefore it will cause the robots to continuously gather information in $\mathcal{D}$.

Proof. For the proof (6) will be used as a common Lyapunov function $V(t)$ for the switching control law (13). Due to the non-stationary problem setup it is only possible to keep the cost function within the stated bounds. In order to achieve that the control law is used to ensure $\ddot{V}(t)<0$ whenever $\dot{V}(t)>0$ holds. As stated before it is sufficient to integrate the control law only over $\mathcal{W}_{i}$ but in order to unify the integration process it is also feasible to integrate over $\mathcal{D}$ because $\mathcal{M}^{\prime}\left(s_{i}\right)$ equals zero outside of $\mathcal{W}_{i}$

But first the stated bounds should be derived. From (5) it is obvious that the lower bound $I_{\min }(\mathbf{p})=0 \forall \mathbf{p} \in \mathcal{D}$ for $I(\mathbf{p}, t)$ exists. Then the maximum difference, and because of A2 therefore also the maximum value of the penalty function $h\left(e_{I}\right)$, is $e_{I}=I_{\text {ref }}-I_{\min }=I_{\text {ref }}$. And because according to A3 - differences in the other direction $\left(e_{I}<0\right)$ are not penalized this is the case in which the penalty function reaches its maximum. Hence the upper bound $C$ for the cost function is $J(t) \leq C=\int_{\mathcal{D}} h\left(I_{\text {ref }}\right) \phi(\mathbf{p}) d \mathbf{p}$. For $N>0$ equality can only be achieved for $t=0$ and an initial information map $I_{0}(\mathbf{p}, 0)=0 \forall \mathbf{p} \in \mathcal{D}$ but not for any $t>0$ because as long as there is at least one robot in $\mathcal{D}$ it will cause the measurement map to be $\mathcal{M}(\mathbf{s})>0$ for some $\overline{\mathbf{p}} \in \mathcal{D}$ and according to (5) this will cause the information map to be $I(\overline{\mathbf{p}}, t)>0$. Hence the value of cost function will be smaller. Since this is a very conservative upper bound which is also not related to the control law, more research will be done on finding a better upper bound $C$. According to A3 the lower bound is $J(t) \geq 0$ for $e_{I} \leq 0$. The lower bound is only reached if there are enough robots s.t. $\mathcal{M}(\mathbf{s}) \geq-\delta I_{\text {ref }} \forall \mathbf{p} \in \mathcal{D}$ holds for all time. This basically means that there are enough robots to keep the measurement map high enough s.t. the information map never drops below the reference information map after it exceeds it for the first time.

The common Lyapunov function and its derivatives will be discussed in the following. The exclamation marks should indicate that these inequalities are not necessarily fulfilled but should be fulfilled in the below stated cases. 


$$
\begin{aligned}
& V(t)=\int_{\mathcal{D}} \underbrace{h\left(e_{I}\right)}_{\geq 0} \underbrace{\phi(\mathbf{p}, t)}_{\geq 0} d \mathbf{p} \geq 0 \\
& \dot{V}(t)=\int_{\mathcal{D}} \underbrace{h^{\prime}\left(e_{I}\right)}_{\geq 0}(\underbrace{-\delta I(\mathbf{p}, t)}_{\geq 0} \underbrace{-\mathcal{M}(\mathbf{s})}_{<0} \underbrace{\phi(\mathbf{p}, t)}_{\geq 0} d \mathbf{p} \\
& +\int_{\mathcal{D}} \underbrace{h\left(e_{I}\right)}_{\geq 0} \underbrace{\frac{\partial \phi}{\partial t}(\mathbf{p}, t)}_{?} d \mathbf{p} \stackrel{!}{\leq} 0 \\
& \ddot{V}(t)=\int_{\mathcal{D}} \underbrace{h^{\prime \prime}\left(e_{I}\right)(-\delta I(\mathbf{p}, t)-\mathcal{M}(\mathbf{s}))^{2} \phi(\mathbf{p}, t)}_{\geq 0} d \mathbf{p} \\
& +\int_{\mathcal{D}} \underbrace{\delta}_{\leq 0} \underbrace{h^{\prime}\left(e_{I}\right)(-\delta I(\mathbf{p}, t)-\mathcal{M}(\mathbf{s})) \phi(\mathbf{p}, t)}_{\geq 0} d \mathbf{p} \\
& +2 \sum_{i \in S} \underbrace{M_{\mathcal{W}_{i}}}_{\leq 0} \underbrace{\left(C_{\mathcal{W}_{i}}-\mathbf{q}_{i}\right) \mathbf{u}_{i}^{*}}_{!} \\
& +2 \int_{\mathcal{D}} \underbrace{h^{\prime}\left(e_{I}\right)}_{\geq 0} \underbrace{(-\delta I(\mathbf{p}, t)-\mathcal{M}(\mathbf{s}))}_{\geq 0} \underbrace{\frac{\partial \phi}{\partial t}(\mathbf{p}, t)}_{?} \\
& +2 \int_{\mathcal{D}} \underbrace{h\left(e_{I}\right)}_{\geq 0} \underbrace{\frac{\partial^{2} \phi}{\partial t^{2}}(\mathbf{p}, t)}_{?} d \mathbf{p} \stackrel{!}{\leq} 0
\end{aligned}
$$

From A2 and A3 it is obvious that inequality (14) is fulfilled with equality iff $e_{I}=I_{r e f}(\mathbf{p})-I(\mathbf{p}, t) \leq 0 \quad \forall \mathbf{p} \in$ $\mathcal{D}$ holds.

But inequality (15) does not necessarily hold. It can be reformulated as follows such that it shows us bounds on $\delta$.

$$
(0 \geq) \delta \geq \frac{-\int_{\mathcal{D}} h^{\prime}(x) \mathcal{M}(s) \phi(\mathbf{p}, t) d \mathbf{p}+\int_{\mathcal{D}} h(x) \frac{\partial \phi}{\partial t}(\mathbf{p}, t) d \mathbf{p}}{\int_{\mathcal{D}} h^{\prime}(x) I(\mathbf{p}, t) \phi(\mathbf{p}, t) d \mathbf{p}}
$$

If this inequality is violated the objective function will start to rise. So the goal is to keep the cost function within bounds around that critical point. To achieve that, it has to be verified that the cost function will decrease again after a certain time. Therefore inequality (16) has to hold in that case. The indicated signs of the terms in (16) are also only necessarily true in that case. Please note that $(-\delta I(\mathbf{p}, t)-\mathcal{M}(\mathbf{p}, t))>0$ also does not necessary hold pointwise but should indicate the sign of the integration result. The input $\mathbf{u}_{i}$ must be chosen s.t. the inequality holds. This can only be achieved with a sufficiently big positive input $\mathbf{u}_{i}$ as indicated in (16). Since we assumed $\frac{\partial \phi}{\partial t}$ and $\frac{\partial^{2} \phi}{\partial t^{2}}$ to be small they were neglected in these thoughts. But it might also be considered as a disturbance and in the future one might look into that problem from the robust control point of view.

But first let $\tilde{S}$ be defined as the arbitrary index set that contains those agents, which use the control law $\mathbf{u}_{i}$, and $\hat{S}$ as the arbitrary index set that contains those agents, which use $\hat{\mathbf{u}}_{i}$. Of course $\tilde{S} \bigcup \hat{S}=S$ and $\tilde{S} \cap \hat{S}=\emptyset$ have to hold. With the use of (10), (12), (13) and $\mathbf{v}_{i}=C_{\mathcal{W}_{i}}-\mathbf{q}_{i}$ the term of (16) which contains the input becomes:

$$
\begin{aligned}
2 \sum_{i \in S} M_{\mathcal{W} i} \mathbf{v}_{i} \mathbf{u}_{i}= & -2 \sum_{i \in \tilde{S}} k_{i} M_{\mathcal{W} i}^{2} \mathbf{v}_{i}^{2} \\
& -2 \sum_{j \in \hat{S}} \hat{k}_{j} M_{\mathcal{W} j} \mathbf{v}_{j}\left(\mathbf{q}_{j}-\hat{\mathbf{p}}_{j}\right)
\end{aligned}
$$

Please note that for all agents $\mathcal{A}_{j}$ with $j \in \hat{S}$ condition (11) holds and thus because of

$$
h^{\prime}\left(e_{I}\right)=0 \stackrel{(8)}{\Rightarrow} M_{\mathcal{W}_{j}}=0 \quad \forall p \in \mathcal{W}_{j} \text { with } j \in \hat{S}
$$

the last term of the equation above is equal to zero and therefor we finally obtain the inequality:

$$
\begin{aligned}
\ddot{V}= & \int_{\mathcal{D}} h^{\prime \prime}\left(e_{I}\right)(-\delta I(\mathbf{p}, t)-\mathcal{M}(\mathbf{s}))^{2} \phi(\mathbf{p}, t) d \mathbf{p} \\
& +\int_{\mathcal{D}} \delta h^{\prime}\left(e_{I}\right)(-\delta I(\mathbf{p}, t)-\mathcal{M}(\mathbf{s})) \phi(\mathbf{p}, t) d \mathbf{p} \\
& -2 \sum_{i \in \tilde{S}} k_{i} M_{\mathcal{W} i}^{2}\left(C_{\mathcal{D} i}-\mathbf{q}_{i}\right)^{2} \\
& +2 \int_{\mathcal{D}} h^{\prime}\left(e_{I}\right)((-\delta I(\mathbf{p}, t)-\mathcal{M}(\mathbf{s}))) \frac{\partial \phi}{\partial t}(\mathbf{p}, t) \\
& +2 \int_{\mathcal{D}} h(x) \frac{\partial^{2} \phi}{\partial t^{2}}(\mathbf{p}, t) d \mathbf{p} \leq 0
\end{aligned}
$$

which holds for sufficiently big $k_{i}$. Therefore the cost function will stay within the desired bounds.

Remark 2. (Discussion of control law $\hat{\mathbf{u}}_{i}\left(\mathbf{q}_{i}\right)$ :)

As obvious from the proof, switching to the second control mode $\hat{\mathbf{u}}_{i}\left(\mathbf{q}_{i}\right)$ does not change the stability argument. But it is immediately clear (by applying simple linear analysis) that this control law will drive the agents $\mathcal{A}_{j}$ to the point $\hat{\mathbf{p}}_{j}$ in infinite time. Though, it is not necessary for the robot to reach that point because as soon as there is a point in his sensory range for which condition (11) does not hold (e.g. $\hat{\mathbf{p}}_{j}$ enters $\mathcal{W}_{j}$ ) it will switch back to the first control law. This shows that (12) drives the agents $\mathcal{A}_{j}$ to a state where (10) will be used again.

We exclude cases with infinite switching here. Future work will address this possible problem.

Partially connected robot group: Now the validity of theorem 1 for partially or even non connected robot groups will be shown. But first a worst case scenario is discussed. Theorem 1 also holds for an index set $S_{1}=1$. This means that there is only one single agent $\mathcal{A}_{1}$ available for covering the area $\mathcal{D}$. Thus theorem 1 holds obviously for a group of disconnected robots. In that case each robot tries to cover $\mathcal{D}$ by himself without recognizing the efforts of the others. Starting from this point the index sets $\hat{S}_{i} \subseteq S$ with $i=1, \ldots, n$ can be defined for $n$ disconnected subgroups with the properties $\bigcup_{i=1}^{n} \hat{S}_{i}=S$ and $\hat{S}_{i} \bigcap_{\substack{i, j \\ i \neq j}} \hat{S}_{j}=\emptyset$. It is assumed that the robots of each group can communicate with all of their group members but not with robots of other groups. Now a group specific objective function $\hat{J}_{i}$ for each group can be defined as

$$
\hat{J}_{i}(t)=\int_{\mathcal{D}} h\left(I_{r e f}(\mathbf{p})-\hat{I}_{i}(\mathbf{p}, t)\right) \phi(\mathbf{p}, t) d \mathbf{p}
$$

with the group specific information map $\hat{I}_{i}(\mathbf{p}, t)$, which also evolves according to equation (5) but with a group specific measurement map $\hat{\mathcal{M}}_{i}\left(s_{i}\right)=\sum_{j \in \hat{S}_{i}} \mathcal{M}_{j}$. Obviously the inequality $\hat{J}_{i}(t) \geq J(t)$ with equality iff $\hat{S}_{i}=S$ holds. The new common Lyapunov function can be defined as

$$
\hat{V}(t)=\sum_{i} \hat{J}_{i}(t)
$$

This will lead to similar expressions as we have obtained in the proof for the fully connected robot group and therefore 
the proof follows exactly the same strategy. This shows us that each robot group for itself tries to cover the points of interest.

\section{SIMULATION}

In this section we will see 3 robots covering a square area $\mathcal{D}$ of 50x50 units length in which a point of interest is moving. The point of interest is modeled by a Gaussian which is moving in a counter-clockwise circular motion around the middle point of the map.

The simulation starts with an initial information map $I(\mathbf{p}, 0)=I_{0}=0 \forall \mathbf{p} \in \mathcal{D}$. As reference information map $I_{r e f}(\mathbf{p})=5 \forall \mathbf{p} \in \mathcal{D}$ is used. The measurement function (4) with the sensor range $r_{i}=2$ and the peak sensing capacity $C_{i}=2$ and the penalty function $h(x)=(\max (0, x))^{2}$ are used. The decay rate and the control gains are set to $\delta=-0.03$ and $k_{i}=\hat{k}_{i}=5$ respectively. For the integrations a simple trapezoidal method is applied.

In fig. 2 the evolution of the cost function over the time is shown. It can be seen that even with the tiny sensor range of $r_{i}=2$ the robots manage to keep the cost function bounded. On the last page the simulation results belonging to the cost function depicted in 2 are shown in two columns. In the left column the time-variant density function $\phi(\mathbf{p}, t)$ is depicted at different time steps. In the right column the corresponding evolution of the information map $I(\mathbf{p}, t)$ can be seen at the same time steps.

It can be seen that the robots create a high level of information always near the peak of the density function and therefore they have to follow its moving peak. There are always 2 robots maintaining the high level of information near this peak and the third robot tries to gather information about other areas ${ }^{1}$.

If we would increase the sensor range, the robots could gather more information and at a certain point all robots would move away from the peak of the density function to gather information somewhere else until the level of information at the peak has dropped sufficiently. Then one or more would move back to the peak.

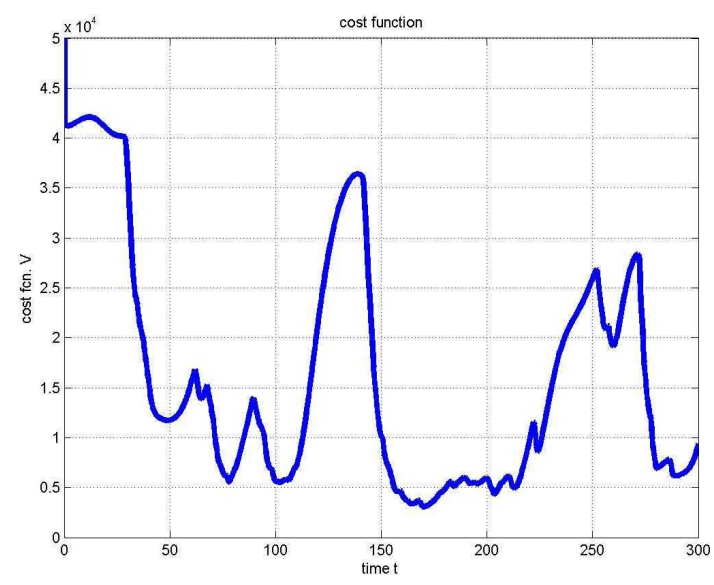

Fig. 2. Cost function

1 There are videos of other simulations available at http : //fujita.fl.ctrl.titech.ac.jp/researches $/ 2007 /$ search/3robots - dot 1 delta,phi $=$ ones.mpg and http : //fujita.fl.ctrl.titech.ac.jp/researches/2007/search/3rob15range_phi45 + 5.mpg.

\section{CONCLUSION}

In this paper we have formulated a coverage control problem with a large variety of applications. We have introduced the novel concept of an information model with information decay in order to derive a control law that - under the given assumptions - causes small groups of robots to gather information about an area without converging to final positions and thus being capable of monitoring an area for infinite time. Additionally this control law can be applied in dynamic environments and a modeling possibility for moving points of interest was described. Then it was shown that with the introduced control law the cost function is bounded and it was pointed out that this control law is also valid for partially connected robot groups with only minor changes. Finally a numerical simulation was presented.

For the future there are several areas of work in addition to the points already mentioned in the paper. Especially collision avoidance, more realistic models of the robots, dynamic network topologys and the use of anisotropic sensors might be interesting. In addition a better cooperation of the robots might be achieved using predictive control methods like MPC.

\section{ACKNOWLEDGEMENTS}

This work was inspired by Hussein [2007] and the authors would like to thank I. Hussein and D.M. Stipanović for this inspiration and the grateful supply of manuscripts.

\section{REFERENCES}

S. Martínes, J. Cortés and F. Bullo. Motion Coordination with Distributed Information. IEEE Control Systems Magazine, pages 75-88, 2007.

J. Cortés, S. Martinez, T. Karatas and F. Bullo. Coverage control for mobile sensing networks. IEEE Transactions on Robotics and Automation, pages 243-255, 2004.

S. Martínes, J. Cortés and F. Bullo Spatially-Distributed Coverage Optimization and Control with LimitedRange Interactions. ESAIM: Control, Optimization and Calculus of Variations, pages 691-719, 2005.

R. Olfati-Saber. Distributed Tracking for Mobile Sensor Networks with Information-Driven Mobility. Proc. of the American Control Conference, 2007.

S. Martínez. On Optimal Sensor Placement and Motion Coordination for Target Tracking. Proc. of the IEEE International Conference on Robotics and Automation, pages 4544-4549, 2005.

A. Ahmadzadeh, A. Jadbabaie, V. Kumar and G. J. Pappas. Cooperative Coverage using Receding Horizon Control. European Control Conference, 2007.

I. I. Hussein and D. M. Stipanović. Effective Coverage Control for Mobile Sensor Networks With Guaranteed Collision Avoidance. IEEE Transactions on Control Systems Technology, Vol. 15, No. 4, July 2007.

O. A. Basir and H.C. Shen. Informational Maneuvering in Dynamic Environment. IEEE International Conference on Systems, Man and Cybernetics, pages 999-1004 vol.2, 1995.

and http: //fujuta.flictri.titech.ac.jp/researches/2007/search/3robl5range_phi45+5.mpg. 

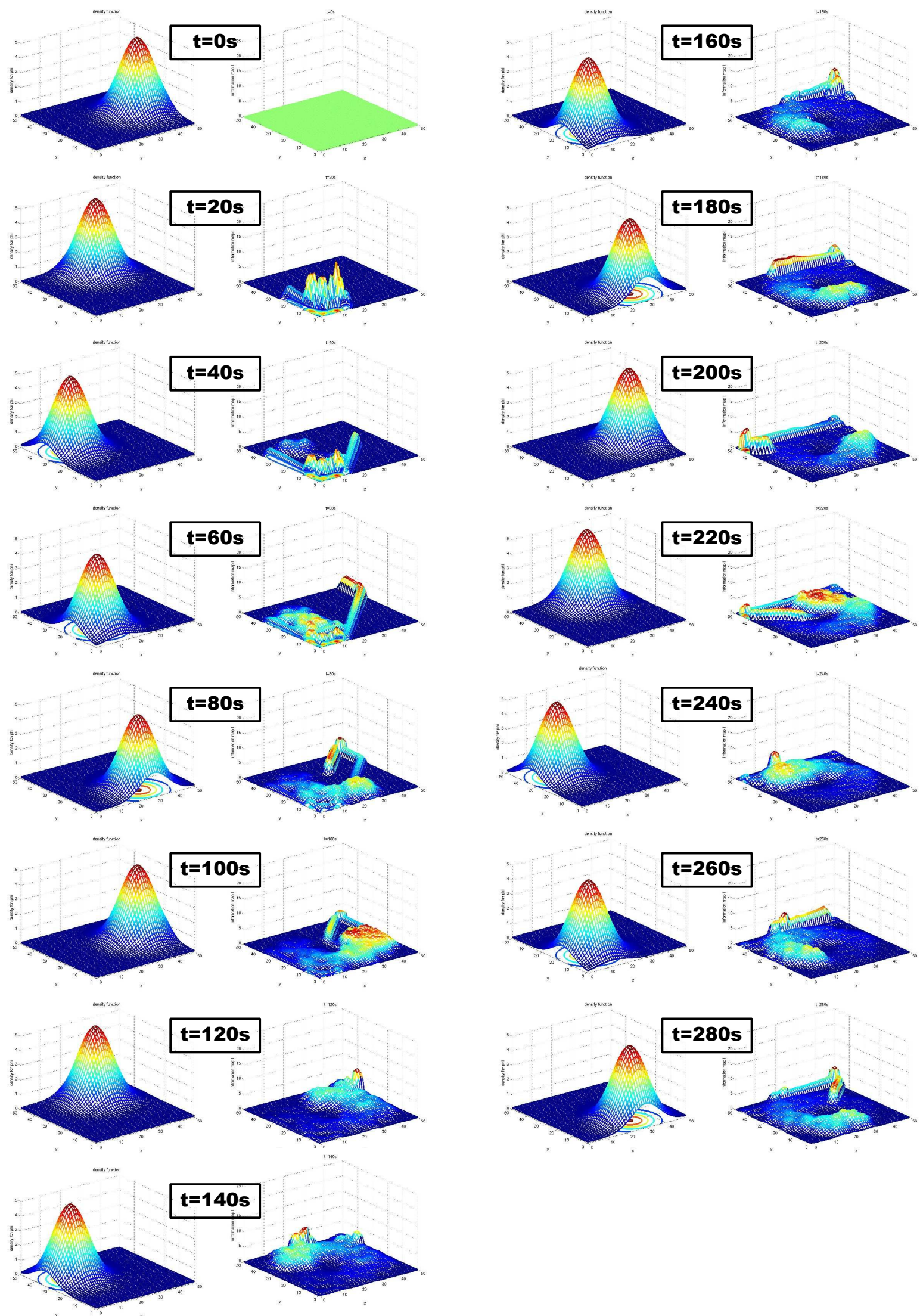

Fig. 3. Simulation: In the left column you can see the time-variant density function $\phi(\mathbf{p}, t)$ whereas in the right column the according evolution of the information map $I(\mathbf{p}, t)$ is depicted. 